\title{
Article \\ Dehydration before Major Urological Surgery and the Perioperative Pattern of Plasma Creatinine: A Prospective Cohort Series
}

\author{
Lukas M. Löffel ${ }^{1}{ }^{(D}$, Dominique A. Engel ${ }^{1}$, Christian M. Beilstein $^{1}{ }^{(D}$, Robert G. Hahn ${ }^{2}$, Marc A. Furrer $^{3}$ \\ and Patrick Y. Wuethrich ${ }^{1, *}$
}

1 Department of Anaesthesiology and Pain Medicine, Inselspital, Bern University Hospital, University of Bern, CH 3010 Bern, Switzerland; lukas.loeffel@insel.ch (L.M.L.); dominique.engel@insel.ch (D.A.E.); christian.beilstein@insel.ch (C.M.B.)

2 Research Unit, Södertälje Hospital, Södertälje, Karolinska Institutet at Danderyds Hospital (KIDS), 18288 Stockholm, Sweden; robert.hahn54@gmail.com

3 Department of Urology, Inselspital, Bern University Hospital, University of Bern, CH 3010 Bern, Switzerland; marcalain.furrer@outlook.com

* Correspondence: patrick.wuethrich@insel.ch; Tel.: +41-31-632-0554

Citation: Löffel, L.M.; Engel, D.A.; Beilstein, C.M.; Hahn, R.G.; Furrer, M.A.; Wuethrich, P.Y. Dehydration before Major Urological Surgery and the Perioperative Pattern of Plasma Creatinine: A Prospective Cohort Series. J. Clin. Med. 2021, 10, 5817. https://doi.org/10.3390/jcm10245817

Academic Editor: Won Ho Kim

Received: 2 October 2021

Accepted: 9 December 2021

Published: 13 December 2021

Publisher's Note: MDPI stays neutral with regard to jurisdictional claims in published maps and institutional affiliations.

Copyright: (c) 2021 by the authors. Licensee MDPI, Basel, Switzerland. This article is an open access article distributed under the terms and conditions of the Creative Commons Attribution (CC BY) license (https:/ / creativecommons.org/licenses/by/ $4.0 /)$.

\begin{abstract}
Preoperative dehydration is usually found in $30-50 \%$ of surgical patients, but the incidence is unknown in the urologic population. We determined the prevalence of preoperative dehydration in major elective urological surgery and studied its association with postoperative outcome, with special attention to plasma creatinine changes. We recruited 187 patients scheduled for major abdominal urological surgery to participate in a single-center study that used the fluid retention index (FRI), which is a composite index of four urinary biomarkers that correlate with renal water conservation, to assess the presence of dehydration. Secondary outcomes were postoperative nausea and vomiting (PONV), return of gastrointestinal function, in-hospital complications, quality of recovery, and plasma creatinine. The proportion of dehydrated patients at surgery was $20.4 \%$. Dehydration did not correlate with quality of recovery, PONV, or other complications, but dehydrated patients showed later defecation $(p=0.02)$ and significant elevations of plasma creatinine after surgery. The elevations were also greater when plasma creatinine had increased rather than decreased during the $24 \mathrm{~h}$ prior to surgery $(p<0.001)$. Overall, the increase in plasma creatinine at $6 \mathrm{~h}$ after surgery correlated well with elevations on postoperative days one and two. In conclusion, we found preoperative dehydration in one-fifth of the patients. Dehydration was associated with delayed defecation and elevated postoperative plasma creatinine. The preoperative plasma creatinine pattern could independently forecast more pronounced increases during the early postoperative period.
\end{abstract}

Keywords: dehydration; gastrointestinal function; major urologic surgery; perioperative plasma creatinine pattern

\section{Introduction}

The degree of preoperative dehydration and its relationship to postoperative outcome is unclear. A particular reported difficulty when studying these issues is obtaining adequate power for statistical significance [1]. The optimal choice of diagnostic remains a matter of debate. A raised serum osmolality is most commonly used to detect a deficit of body water, while concentrated urine appears to be a simpler and even more sensitive alternative [2-4]. The reported incidence of preoperative dehydration, when based on urine sampling, is approximately one-third to half of all patients in different surgical populations [5-7]. The prevalence in the urologic population is not presently known, but it is relevant because an adequate fluid regime in the perioperative period might reduce complications [8,9]. Some studies show that preoperative dehydration increases the risk of overall postoperative complications, while others indicate a negative effect on morbidity, for example, by causing 
postoperative nausea and vomiting (PONV) [1,5]. Other studies have been unable to show these effects [10].

The objective of this observational study was to determine the prevalence of preoperative dehydration, as measured by the fluid retention index (FRI), among candidates for elective major urologic surgery and its relationship to postoperative outcome. FRI is a summary measure of several indices of concentrated urine that aims to provide a robust assessment of renal water conservation [10]. We collected data on the incidence of surgery-associated nausea and vomiting, postoperative return of gastrointestinal function, fluid balance, complications within hospitalization, quality of recovery, and renal function. Special emphasis was placed on the perioperative pattern of plasma creatinine.

\section{Materials and Methods}

\subsection{Approvals}

This monocentric observational study reports a single tertiary center cohort series, and accords with the Strengthening the Reporting of Observational Studies in Epidemiology (STROBE) recommendations. Ethical approval was provided by the Ethics Committee of the Canton Bern, Switzerland (KEK Bern, Project-ID 2018-01804, Chairperson Professor C. Seiler) on 3 December 2018, and the study was registered at ClinicalTrials.gov (accessed on 21 December 2018) with the identifier NCT03788070. Written informed consent was obtained from all participants.

\subsection{Study Population and Design}

The study goal was to consecutively identify and include patients at the Department of Urology of the University Hospital Bern undergoing major abdominal urologic surgery. Inclusion started on 11 February 2019 and ended on 16 March 2020 (due to the COVID-19 crisis), and comprised patients scheduled for elective major urologic laparotomy or robotic assisted laparoscopy, which included prostatectomy, bladder and kidney surgery, reconstruction of the ureter, and open retroperitoneal lymph node dissection. Exclusion criteria were preoperative need for dialysis, preoperative IV fluid administration, age $<18$ years, pregnancy, and enrollment of employees and other dependent persons.

\subsection{Outcome Measures}

The primary outcome was the prevalence of dehydration before the surgery, as defined by the fluid retention index (FRI), which is the mean score of four urine measurements that reflect renal water conservation, considered consistent with dehydration. A patient is deemed dehydrated if the FRI is $\geq 4$.0; this cut-off corresponds to a urine-specific gravity of $\geq 1.020$, urine osmolality $\geq 600 \mathrm{mosmol} / \mathrm{kg}$, urine creatinine $\geq 12 \mathrm{mmol} / \mathrm{L}$, and urine color $\geq 4$, as assessed visually on a standardized color chart (Table 1) $[4,7,11]$.

Table 1. Description of the four dimensions of the fluid retention index (FRI).

\begin{tabular}{ccccccc}
\hline Score & $\mathbf{1}$ & $\mathbf{2}$ & $\mathbf{3}$ & $\mathbf{4}$ & $\mathbf{5}$ & $\mathbf{6}$ \\
\hline Specific gravity & $\leq 1.005$ & 1.010 & 1.015 & 1.020 & 1.025 & 1.030 \\
Osmolality (mOsmol/kg) & $<250$ & $250-450$ & $450-600$ & $600-800$ & $800-1000$ & $>1000$ \\
Creatinine (mmol/L) & $<4$ & $4-7$ & $7-12$ & $12-17$ & $17-25$ & $>25$ \\
Color (shade) & 1 & 2 & 3 & 4 & 5 & 6 \\
\hline
\end{tabular}

Secondary outcomes were PONV, return of gastrointestinal function, intra- and postoperative fluid balance, complications during hospitalization (Appendix A), quality of recovery judged by the quality of recovery-15 (QoR-15) questionnaire, and postoperative renal function, as indicated by repeated measurements of plasma creatinine [12].

\subsection{Data Collection and Laboratory Tests}

Urine and plasma samples were collected the day before surgery (at around 2 p.m.; time point defined as "preoperative"), at the start of surgery (directly after induction of 
anesthesia, time point defined as "surgery"), $6 \mathrm{~h}$ after surgery ended, and at $6 \mathrm{a} . \mathrm{m}$. on postoperative day one (POD1) and postoperative day two (POD2). Urine samples were taken directly from the ureteral catheters if present (bladder and kidney surgery) or from the urethral catheter.

Urine samples were analyzed for specific gravity, osmolality, creatinine, and color. The plasma was analyzed for osmolality, creatinine, sodium, potassium, hemoglobin, and hematocrit. The measurements were performed by the certified clinical chemistry laboratory at our hospital (osmolality: Station 6060 TT, Array Global Business Inc., Japan, and urine/plasma electrolytes: COBAS 8000/ISE Modul, Roche Diagnostics, Basel, Switzerland). Urine specific gravity was determined with a digital photometric refractometer, which expresses values in steps of 0.005 based on the reflection coefficient of light directed onto strips dipped in urine (Uricon-Ne, Atago CO. LTD., Tokyo, Japan).

A dedicated study nurse assessed PONV, based on a verbal rating scale and emetic episodes, at the same time as laboratory samples were drawn. Data on onset of flatus, defecation, and the quality of recovery (QoR 15 questionnaire) were also obtained. Intraoperative fluid administration and blood loss, fluid balance (difference in body weight between preoperative and POD1), and complications during hospitalization (Bennett Guerrero score) were assessed from the hospital medical records [13].

\subsection{Perioperative Management}

Pre-operative oral hydration including carbohydrate-loading beverages was allowed up to $2 \mathrm{~h}$ before the start of anesthesia. No enteral bowel preparation and no enemas were performed preoperatively. Patients were allowed to drink clear fluids immediately after surgery while in the intermediate care unit. An oral liquid diet was started on POD1. Perioperative patient management was based on an Enhanced Recovery Approach [14,15]. PONV prophylaxis was based on preoperative Apfel score assessment, and performed in accordance with the consensus recommendation [16,17].

Surgery was performed under general anesthesia. After induction with propofol (1-2 mg/ $\mathrm{kg})$, rocuronium $(0.6-0.9 \mathrm{mg} / \mathrm{kg})$, and fentanyl $(2 \mu \mathrm{g} / \mathrm{kg})$, patients were ventilated with tidal volumes of $6 \mathrm{~mL} / \mathrm{kg}$ ideal body weight and a positive end-expiratory pressure (PEEP) of $5-10 \mathrm{mmHg}$, depending on body mass index (BMI). Baseline plasma volume support consisted of fluid administration of Ringer's lactate at a rate up to $4 \mathrm{~mL} / \mathrm{kg} / \mathrm{h}$. If hypotension was observed (mean arterial pressure $<60 \mathrm{mmHg}$ ), norepinephrine was titrated to a maximum of $8 \mu \mathrm{g} / \mathrm{kg} / \mathrm{h}$ after an initial bolus of 5-10 $\mu \mathrm{g}$. A fluid bolus of $250 \mathrm{~mL}$ of lactated Ringer's was administered in cases where the patient was judged to be hypovolemic, based on pulse pressure variation $>10 \%[8,18]$.

Blood loss was obtained as the sum of the volume present in the suction bag and the weight of sponges and swabs. Hemorrhage up to $500 \mathrm{~mL}$ was replaced with an equal amount of Ringer's lactate. Packed red blood cells were transfused if blood hemoglobin fell below $80 \mathrm{~g} / \mathrm{L}(<100 \mathrm{~g} / \mathrm{L}$ in patients with severe coronary artery disease). No hydroxyethyl starch solution was given.

\subsection{Statistical Analysis}

Continuous variables were presented as mean \pm standard deviation if normally distributed. Variables with a skewed distribution were reported as the median (25-75\% interquartile range, IQR). Categories were presented as numbers and percentages.

A binomial test and confidence interval were performed to assess the primary outcome at surgery. Secondary outcomes were assessed longitudinally with a Brunner and Langer non-parametric repeated-measures ANOVA, followed by Mann-Whitney tests post hoc [19]. Group-wise comparisons of secondary outcomes were assessed using MannWhitney and Fisher's exact tests. $p<0.05$ was considered statistically significant. Relationships between creatinine values at different time points were evaluated by correlation analysis. A two-way ANOVA, based on $(1+\log )$ transformed values due to skewed 
distribution, was performed for point-wise analysis of dehydration and the preoperative change in plasma creatinine.

All $p$-values for post hoc tests of secondary outcomes and in the retrospective analysis of creatinine ratios were corrected by the Holm method. All analyses in this report were performed with the statistics software R, version 3.5.0.

No formal power analysis was performed, as the incidence and severity of dehydration in patients undergoing major urological surgery was still unknown when the study was initiated. However, based on other types of surgery, we expected to recruit approximately 200 patients (with a variance of $\pm 10 \%$ ) [5].

\section{Results}

We screened 238 consecutive patients scheduled for elective major urological surgery. We initially recruited 193 patients, but five dropped out before the study began (three patients due to cancellation of the surgery, one patient for logistical reasons, and one patient for preoperative dialysis). One patient withdrew consent after inclusion, leaving 187 to provide data. Overall, six patients $(3.2 \%)$ had missing values for the assessment of our primary outcome due to logistical reasons, resulting in 181 patients included in the final analysis of dehydration as the primary endpoint (Supplementary Materials Figure S1).

\subsection{Dehydration}

The proportion of dehydrated patients at surgery was $20.4 \%$ (37/181 patients, $95 \%$ CI: $14.8-27.1 \%)$. A larger fraction of the prostate surgery patients were dehydrated (40.5\%; $p=0.005)$, but other differences in demographics, preoperative health status, and chronic medication were only occasional and slight between hydrated and dehydrated patients (Table 2). The two groups received similar amounts of fluid during the surgeries, with an overall volume of administered crystalloid of $1.3(1.1-2.1) \mathrm{L}$, infused at a rate of $4.5(3.0-6.5) \mathrm{mL} / \mathrm{kg} / \mathrm{h}$.

Table 2. Patient's pre-, intra-, and postoperative data. Dehydration grouping according to fluid retention index (FRI) $\geq 4$ obtained when surgery was initiated. Data shown as median (25th-75th percentiles) or block column percentages depending on distribution. ${ }^{+}$values measured at surgery. Missing data coming from patients in the hydrated group.

\begin{tabular}{|c|c|c|c|c|}
\hline & All Patients $n=181$ & Dehydrated $n=37$ & Hydrated $n=144$ & $p$-Value \\
\hline \multicolumn{5}{|l|}{ Type of Surgery } \\
\hline Prostate $n(\%)$ & $44(24.3 \%)$ & $15(40.5 \%)$ & $29(20.1 \%)$ & 0.005 \\
\hline Bladder $n(\%)$ & $50(27.6 \%)$ & $3(8.1 \%)$ & $47(32.6 \%)$ & \\
\hline Kidney $n(\%)$ & $51(28.2 \%)$ & $11(29.7 \%)$ & $40(27.8 \%)$ & \\
\hline Other $n(\%)$ & $36(19.9 \%)$ & $8(21.6 \%)$ & $28(19.4 \%)$ & \\
\hline Open/minimal invasive $n(\%)$ & $130(72 \%) / 51(28 \%)$ & $23(62 \%) / 14(38 \%)$ & $107(74 \%) / 37(26 \%)$ & 0.22 \\
\hline Age (years) & $65.0(56.5-71.0)$ & $62.0(51.0-68.0)$ & $65.5(56.8-72.0)$ & 0.04 \\
\hline BMI (kg/height²) & $26.9(23.9-29.7)$ & $29.0(24.2-32.5)$ & $26.6(23.9-29.1)$ & 0.04 \\
\hline ASA $1 n(\%)$ & $9(5 \%)$ & $3(8.1 \%)$ & $6(4.2 \%)$ & 0.16 \\
\hline ASA $2 n(\%)$ & $81(44.8 \%)$ & $21(56.8 \%)$ & $60(41.7 \%)$ & \\
\hline ASA $3 n(\%)$ & $89(49.2 \%)$ & $13(35.1 \%)$ & $76(52.8 \%)$ & \\
\hline ASA $4 n(\%)$ & $2(1.1 \%)$ & $0(0.0 \%)$ & $2(1.4 \%)$ & \\
\hline Ischemic heart disease $n(\%)$ & $26(14.5 \%)$ & $3(8.3 \%)$ & $23(16.1 \%)^{* *}$ & 0.30 \\
\hline Hypertension $n(\%)$ & $86(47.5 \%)$ & $19(51.4 \%)$ & $67(46.5 \%)$ & 0.71 \\
\hline Diabetes mellitus $n(\%)$ & $18(9.9 \%)$ & $5(13.5 \%)$ & $13(9.0 \%)$ & 0.54 \\
\hline Smoking $n(\%)$ & $54(31 \%)$ & $17(45.9 \%)$ & $37(25.6 \%)$ & 0.03 \\
\hline $\mathrm{GFR}>90 \mathrm{~mL} / \mathrm{min}$ & $71(39.7 \%)$ & $21(56.8 \%)$ & $50(35.2 \%)^{* *}$ & $<0.0001$ \\
\hline GFR $60-89 \mathrm{~mL} / \mathrm{min}$ & $63(35.2 \%)$ & $16(43.2 \%)$ & $47(33.1 \%)$ & \\
\hline $\mathrm{GFR}<60 \mathrm{~mL} / \mathrm{min}$ & $45(25.1 \%)$ & $0(0.0 \%)$ & $45(31.7 \%)$ & \\
\hline Betablocker $n(\%)$ & $29(16.2 \%)$ & $7(18.9 \%)$ & $22(15.5 \%) * *$ & 0.62 \\
\hline Calcium antagonists $n(\%)$ & $79(43.6 \%)$ & $1643.2 \%)$ & $63(43.8 \%)$ & 1.00 \\
\hline Statins $n(\%)$ & $44(24.3 \%)$ & $12(32.4 \%)$ & $32(22.2 \%)$ & 0.20 \\
\hline Aspirin $n(\%)$ & $28(15.6 \%)$ & $8(21.6 \%)$ & $20(14.0 \%)$ & 0.31 \\
\hline Diuretics $n(\%)$ & $17(9.4 \%)$ & $2(5.4 \%)$ & $15(10.5 \%)^{* *}$ & 0.53 \\
\hline Antidiabetics $n(\%)$ & $17(9.6 \%)$ & $5(13.5 \%)$ & $12(8.5 \%) * *$ & 0.35 \\
\hline Hemoglobin $^{+}(\mathrm{mmol} / \mathrm{L})$ & $135(122-144)$ & $140(131-144)$ & $133(118-143)$ ** & 0.01 \\
\hline Osmolality ${ }^{+}$(mosmol/kg) & $288(284-291)$ & $288(285-290)$ & $288(283-291) * *$ & 0.65 \\
\hline Creatinine $^{+}(\mu \mathrm{mol} / \mathrm{L})$ & $84(70-96)$ & $73(65-85)$ & $85.5(72-102) *$ & 0.004 \\
\hline
\end{tabular}


Table 2. Cont.

\begin{tabular}{|c|c|c|c|c|}
\hline & All Patients $n=181$ & Dehydrated $n=37$ & Hydrated $n=144$ & $p$-Value \\
\hline Intraoperative Ringer lactated (mL) & $1300(1050-2100)$ & $1800(1100-2100)$ & $1300(1025-2100)$ & 0.35 \\
\hline Intraoperative Colloids $(\mathrm{mL})$ & $0(0-0)$ & $0(0-0)$ & $0(0-0)$ & 0.29 \\
\hline Intraoperative packed red blood cells (mL) & $0(0-0)$ & $0(0-0)$ & $0(0-0)$ & 0.34 \\
\hline Intraoperative fresh frozen plasma $(\mathrm{mL})$ & $0(0-0)$ & $0(0-0)$ & $0(0-0)$ & 0.21 \\
\hline Blood Loss $(\mathrm{mL})$ & $400(200-700)$ & $400(200-600)$ & $400(200-750)$ & 0.90 \\
\hline Fluid balance on -POD1 (kg) & $0.8(-2.2-0.1)$ & $1.0(-2.7-0.0)$ & $0.7(-2.0-0.2)$ & 0.35 \\
\hline
\end{tabular}

$p$-values calculated using exact Fisher or exact Mann-Whitney Tests, depending on data distribution. Number of missing data: ** $n=2$; $* n=1$.

The outcome data showed no statistically significant differences between the groups for blood loss, fluid balance on POD1, in-hospital complications, and the quality of recovery (QoR-15 questionnaire) (Table 3). No differences were noted in the occurrence of flatus $(p=0.48)$ and defecation $(p=0.61)$; however, defecation occurred later in the dehydrated patients $(p=0.02)$. Twice as many dehydrated patients had PONV on POD1 $(22 \%$ vs. $11 \%)$, but the difference over time was not significant $(p=0.48$; Table 4$)$.

Table 3. Non-longitudinal secondary outcomes: Dehydration according to the fluid retention index (FRI) $\geq 4$. Data are presented median (25th-75th percentiles) for numeric outcomes and percentages (95\% CI) for categorical/dichotomous outcomes.

\begin{tabular}{|c|c|c|c|c|}
\hline & $\begin{array}{l}\text { All Patients } \\
\quad n=181\end{array}$ & $\begin{array}{c}\text { Dehydrated } \\
\quad n=37\end{array}$ & $\begin{array}{c}\text { Hydrated } \\
n=144\end{array}$ & $p$-Value \\
\hline \multicolumn{5}{|l|}{ Difference in QoR15 Scores } \\
\hline A-Part (questions 1-10) & $-25(-34.0,-15.0)$ & $-26(-33.5,-16.0)$ & $24.5(-35.8,-14.0)$ & 0.68 \\
\hline B-Part (questions 11-15) & $-1.0(-6.0,2.0)$ & $-2.0(-5.0,0.5)$ & $-1.0(-6.8,2.8)$ & 0.76 \\
\hline Overall (questions 1-15) & $-27.0(-38.0,-14.0)$ & $-28.0(-36.0,-17.0)$ & $-25.5(-38.8,-13.0)$ & 0.55 \\
\hline \multicolumn{5}{|l|}{ In-hospital complications } \\
\hline Bennett-Guerrero Score & $1.0(0.0,1.0)$ & $1.0(0.0,1.0)$ & $1.0(0.0,1.0)$ & 0.18 \\
\hline Cardiovascular Complications (\%) & $5.0(2.3,9.2)$ & $2.7(0.1,14.2)$ & $5.6(2.4,10.7)$ & 0.69 \\
\hline Pulmonary Complications (\%) & $2.8(0.9,6.3)$ & $2.7(0.1,14.2)$ & $2.8(0.8,7.0)$ & 1.00 \\
\hline Infections $(\%)$ & $11.6(7.3,17.2)$ & $5.4(0.7,18.2)$ & $13.2(8.1,19.8)$ & 0.26 \\
\hline Neurological Complications (\%) & $3.9(1.6,7.8)$ & $0.0(0.0,9.5)$ & $4.9(2.0,9.8)$ & 0.35 \\
\hline Renal Complications (\%) & $20.4(14.8,27.1)$ & $18.9(8.0,35.2)$ & $20.8(14.5,28.4)$ & 1.00 \\
\hline Gastrointestinal Complications (\%) & $9.4(5.6,14.6)$ & $2.7(0.1,14.2)$ & $11.1(6.5,17.4)$ & 0.20 \\
\hline Postoperative Transfusion (\%) & $7.7(4.3,12.6)$ & $0.0(0.0,9.5)$ & $9.7(5.4,15.8)$ & 0.08 \\
\hline Deaths $(\%)$ & $0.6(0.0,3.0)$ & $0.0(0.0,9.5)$ & $0.7(0.0,3.8)$ & 1.00 \\
\hline
\end{tabular}

$p$-values were calculated using exact Fisher or exact Mann-Whitney tests, depending on data distribution.

Table 4. Longitudinal secondary outcomes at $6 \mathrm{~h}$ postoperatively on POD 1 and 2.

\begin{tabular}{ccccc}
\hline Variable & Group & 6h postop. & POD 1 & POD 2 \\
\hline PONV (\%) & Dehydrated & $16.2(6.2,32)$ & $21.6(9.8,38.2)$ & $8.1(1.7,21.9)$ \\
& Hydrated & $15.3(9.8,22.2)$ & $11.1(6.5,17.4)$ & $10.5(5.9,16.6)$ \\
Flatus (hours) & Dehydrated & - & $43.2(27.1,60.5)$ & $91.9(78.1,98.3)$ \\
& Hydrated & - & $47.6(38.9,55.7)$ & $79.4(70.1,84.3)$ \\
Defecation * $(\%)$ & Dehydrated & - & $2.7(0.1,14.2)$ & $56.8(39.5,72.9)$ \\
& Hydrated & - & $11.1(6.5,17.4)$ & $43.0(34.2,50.9)$ \\
& & - & $p=0.29$ & $p=0.29$ \\
\hline
\end{tabular}

* significant group factor or interaction in ANOVA including post hoc tests. Data are median (25th-75th percentiles) for numeric outcomes and percentages $(95 \%-\mathrm{CI})$ for categorical/dichotomous outcomes.

The perioperative increase in plasma creatinine was also greater in the dehydrated than in the hydrated patients (Table 5, top).

\subsection{Trajectory of Plasma Creatinine}

The preoperative plasma creatinine concentration was $81(68-90) \mu \mathrm{mol} / \mathrm{L}$. The time course showed that the final postoperative nadir value could be forecasted from the measurement performed at $6 \mathrm{~h}$ after surgery, but patients experiencing a rise in plasma 
creatinine between the preoperative measurement and the sample taken just before surgery started were more likely to have a greater postoperative elevation of plasma creatinine (Table 5, lower section).

Table 5. Statistical comparison between postoperative plasma creatinine relative to the preoperative concentration, depending on the presence of dehydration (as indicated by a preoperative urine sample) and whether plasma creatinine increased or decreased during the preoperative period.

\begin{tabular}{cccc}
\hline $\begin{array}{c}\text { Plasma Creatinine } \\
\text { (\% Change) }\end{array}$ & $\begin{array}{c}\text { Dehydrated } \\
(\boldsymbol{n}=\mathbf{3 7})\end{array}$ & $\begin{array}{c}\text { Hydrated } \\
(\boldsymbol{n}=\mathbf{1 4 4})\end{array}$ & $\boldsymbol{p}$-Value \\
\hline 6 h/preop & $20(13-41)$ & $13(1-25)$ & 0.01 \\
POD 1/preop & $23(9-39)$ & $9(-3-25)$ & 0.02 \\
POD 2/preop & $12(-2-42)$ & $3(-9-18)$ & 0.013 \\
\hline \multicolumn{4}{c}{ Preoperative change in P-creatinine (\% change) } \\
$6 \mathrm{~h}$ /preop & $\geq 1.0(n=82)$ & $<1.0(n=102)$ & 0.001 \\
POD 1/preop & $21(13-36)$ & $8(-6-20)$ & 0.001 \\
POD 2/preop & $18(9-39)$ & $6(-8-21)$ & 0.001 \\
\hline
\end{tabular}

Data are the median and interquartile range. The Mann-Whitney $U$ test was used for statistics.

The correlations suggest that $30 \%$ of the variability in plasma creatinine at $6 \mathrm{~h}$ after surgery could be accounted for by the preoperative change (Figure $1 \mathrm{~A}$ ), and that $83 \%$ of the variability on POD1 could be explained by the change that had occurred by $6 \mathrm{~h}$ (Figure 1B).

\section{Change in plasma creatinine (\%)}
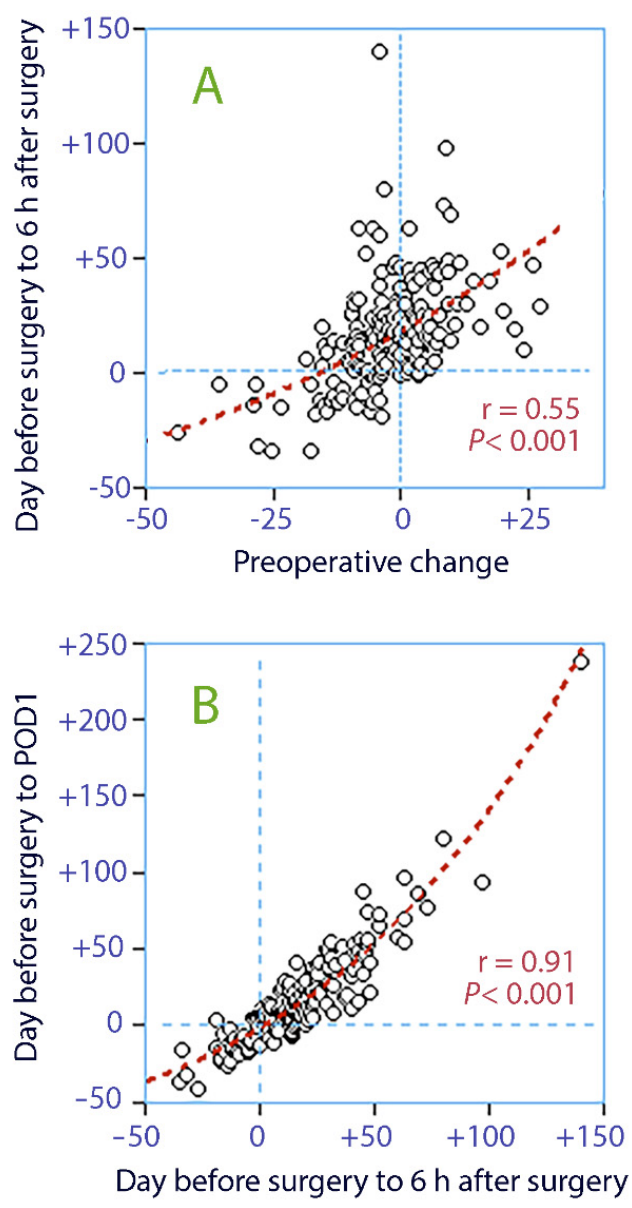

Figure 1. Linear correlations between changes in plasma creatinine; (A) the preoperative change vs. the change from the day before surgery to $6 \mathrm{~h}$ after surgery; (B) the further change to the first postoperative day (POD1). 
Approximately two-thirds of the patients had a reduction in plasma creatinine between POD1 and POD2. The patients with the greatest increase on POD1 showed a further increase, while those with a less pronounced increase had a decrease (difference, $p<0.003$; Figure 2).

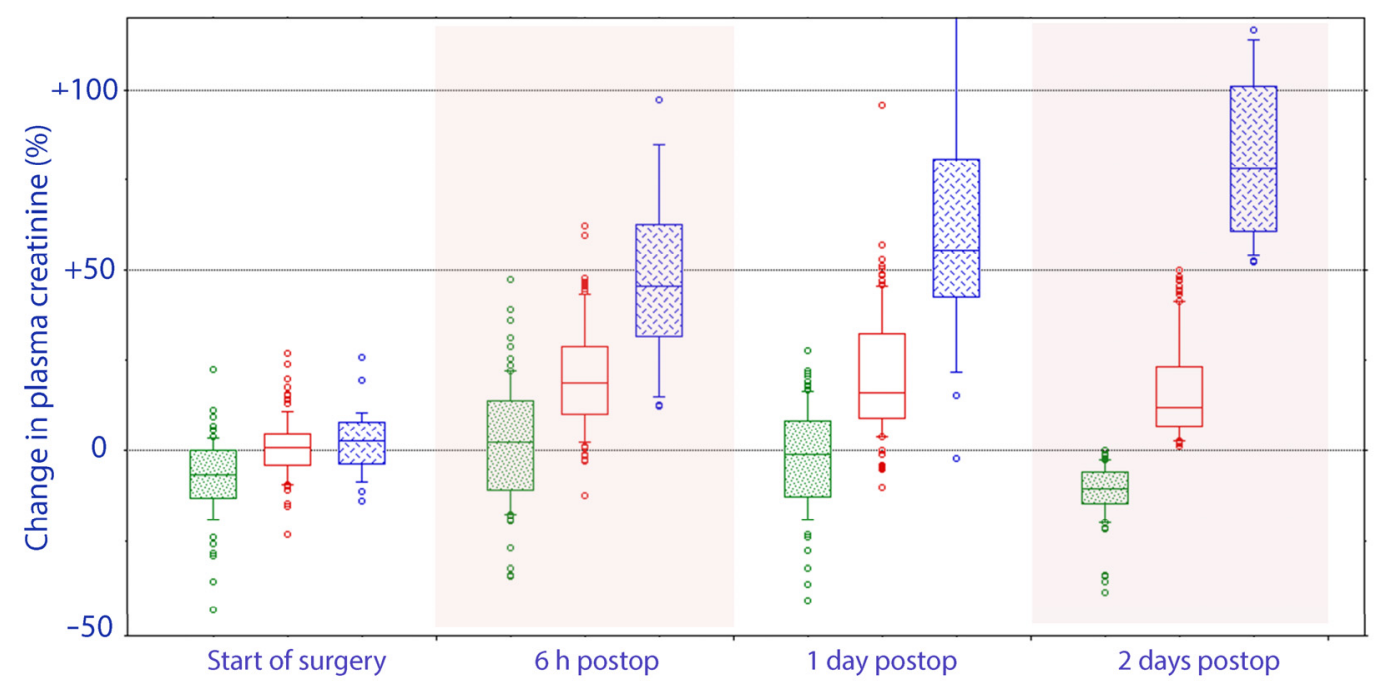

Figure 2. Perioperative trajectory of plasma creatinine based on whether the final postoperative value represented a decrease in plasma creatinine values (green), a moderate increase $(0-50 \%$; red) or a substantial increase ( $>50 \%$, blue) as compared to the concentration measured on the day before surgery. A statistically significant difference between these groups had occurred already before surgery was initiated, i.e., from the day before the surgery to the onset of surgery.

\subsection{Risk Factors for Plasma Creatinine Elevation}

Two-way analysis of variance showed that both dehydration and the preoperative change in plasma creatinine served as statistically significant and independent predictors of the elevation of plasma creatinine at $6 \mathrm{~h}(p<0.05$ and $p<0.001$, respectively) and on POD1 $(p<0.012$ and $p<0.001)$, while only the preoperative change in plasma creatinine was significant on POD2 $(p=0.006)$.

The median rise in plasma creatinine never exceeded $5 \%$ for the group of 82 patients who were both well hydrated and showed a preoperative reduction in plasma creatinine. By contrast, the 18 patients who were both dehydrated and had a preoperative rise in plasma creatinine showed a median increase of $26 \%$ on POD1, and seven had elevations of $40 \%$ or more (Figure 3 ).

Exploratory analyses suggested that a body mass index $>30 \mathrm{~kg} / \mathrm{m}^{2}(n=43)$ served as an additional independent predictor that would strengthen the presented correlations. However, we considered that the subgroups would then be too small for presentation. 


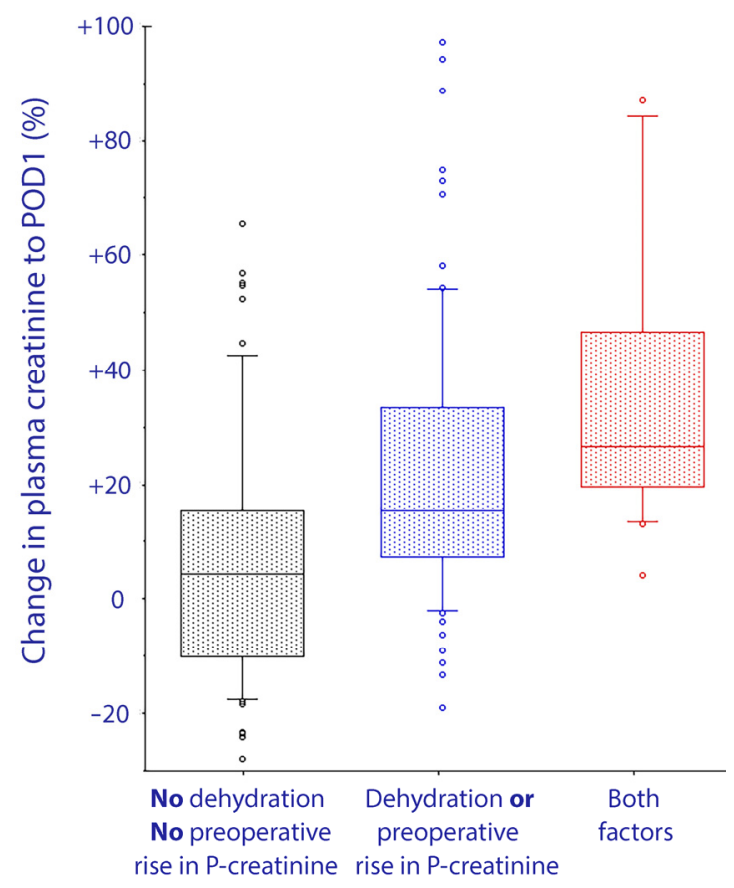

Figure 3. Change in plasma creatinine (as a percentage) from the day before surgery to the first day after surgery, depending on whether patients were dehydrated and/or showed an increase in plasma creatinine from the day before surgery to just before the surgery started. The box shows the 25th, 50th, and 75th percentiles and the error bars indicate the 10th and 90th percentiles.

\section{Discussion}

\subsection{Key Results}

The proportion of dehydrated patients was $20.4 \%$, which was lower than expected, as earlier studies reported a prevalence of 30-50\% [5-7]. Preoperative dehydration was not significantly associated with PONV, fluid balance, or quality of recovery score (QoR15), but dehydrated patients were prone to delayed defecation and postoperative elevations of plasma creatinine. Interestingly, our study of the trajectory showed that the changes in plasma creatinine that occurred while awaiting surgery were also associated with later changes. To our knowledge, this observation has not been reported previously. Preoperative dehydration and this early change in plasma creatinine both appeared to serve as independent risk factors for postoperative elevations of plasma creatinine. Their strength can be illustrated by the fact that one-third of those who presented with dehydration and a $24 \mathrm{~h}$ rise in plasma creatinine had a later postoperative increase of $40 \%$ or more. By contrast, elevations of $5-10 \%$ were rare in patients who did not present with these characteristics.

\subsection{Interpretation}

The later defecation in the dehydrated patients is probably explained by more enteral water resorption and a consequently reduced amount of feces of harder consistency. This agrees with previous findings of a general statistical link between dehydration and obstipation [20].

The dehydration diagnosis we used implies that the kidneys were set to conserve water when surgery began. The additional renal fluid retention induced by anesthesia and surgery could then be troublesome for creatinine excretion. The maximum renal capacity for creatinine excretion is not precisely known, but is likely to vary among individuals.

We hypothesize that fluid retention caused by preoperative stress surpassed the renal threshold for creatinine excretion in those who showed a rise in plasma creatinine before the surgery [21]. These patients probably had a reduced capacity to excrete creatinine due to pre-existing limitations of kidney function. By contrast, dehydration had a less 
pronounced effect on postoperative plasma creatinine in patients who did not show this rise before the surgery.

\subsection{Literature}

Several forms of dehydration are recognized, but the patients diagnosed as being dehydrated in the present study had most certainly ingested very little water just before surgery or had a habitually low intake of water (intracellular dehydration) [2,21]. Chronic dehydration is a challenging diagnosis, and a universally accepted standard reference biomarker is still lacking. Serum osmolality is often recommended; however, hyperosmolality seems to require that the kidneys fail to concentrate the urine [22]. We used urine analysis for the present study, as it has been well evaluated in sports medicine and occasionally applied in hospital care [3,7].

Concentrated urine is common in the general population. Only a few studies have suggested that (chronic) dehydration has an impact on health outcomes [11,23]. Concentrated urine is associated with a high 30-day mortality in acute geriatric care and a higher rate of complications after hip fracture surgery $[5,24]$. Concentrated urine changes the body's handling of both crystalloids and colloids in the sense that more fluid is needed to perform fluid optimization before surgery $[6,25,26]$. In addition, treatment of dehydration if enteral fluid administration is not sufficient or possible (i.e., early postoperative period), the administration of hypotonic solution (glucose $5 \%$ for example) is of importance. Salt load has been also presented as a risk factor for fluid accumulation during the early postoperative phase [27].

Postoperative acute kidney injury (AKI) is a postoperative complication most commonly diagnosed based on a perioperative rise in plasma creatinine. The current criteria (kidney disease: Improving Global Outcomes: KDIGO) for Stage 1 AKI are fulfilled if the plasma creatinine increases by $50 \%$, compared to the baseline [28]. The medical community has shown great concern regarding this complication, since chronic kidney injury occasionally ensues and increases morbidity and mortality $[29,30]$.

\subsection{Future Views}

The mechanisms we suggest do not rest on the occurrence of perioperative harm to the kidneys, but on normal physiological responses to low fluid intake, perioperative stress, and minor pre-existing limitations of renal function. Nevertheless, our findings could raise the possibility of perioperative management in high-risk patients that would predict the risk and possibly prevent substantial postoperative increases in plasma creatinine, and perhaps even acute kidney injury, through urine analysis and preoperative measurement of plasma creatinine.

\subsection{Strengths and Limitations}

One strength of this study is that we included a large sequential cohort of major abdominal urological surgery patients over a short time period. All patients were managed pre-, intra-, and post-operatively in a nearly identical manner, as the perioperative pathway is standardized according to the type of surgery in our institution [8,18]. A limitation of this study was its prospective assessment of data from a single high caseload center, as this could limit the possibility of generalizing the findings. Another possible limitation is that we did not assess neutrophil gelatinase-associated lipocalin (NGAL) perioperatively, and the postoperative increase in plasma creatinine has been shown to be preceded by an elevation of NGAL. However, NGAL elevation itself was linked to the high fluid retention index, suggesting dehydration or mechanisms inducing fluid retention [7].

An additional limitation is the lack of precise hemodynamic information, since we are not able to exclude variations in cardiac output (i.e., low flow situations) during the observation period, despite adequate (invasive arterial blood pressure) monitoring and standard procedures to combat hypovolemia. However, the expectation is that all patients would have been treated the same way (with additional fluid administration and 
vasopressors) to combat hypotension and hypovolemia, as both are known risk factors for acute kidney injury [31].

\section{Conclusions}

Preoperative dehydration was present in one-fifth of the patients. Dehydration was not associated with postoperative complications, PONV or differences in quality of recovery assessed by the QoR15. However, dehydrated patients were prone to delayed defecation and a postoperative rise in plasma creatinine. Even greater elevations of plasma creatinine occurred in patients who also showed a rise in plasma creatinine during the $24 \mathrm{~h}$ prior to the operation.

Supplementary Materials: The following are available online at https: / www.mdpi.com/article / $10.3390 /$ jcm10245817/s1, Figure S1: Flow chart with inclusion and exclusion criteria used in the analysis.

Author Contributions: L.M.L., R.G.H. and P.Y.W. contributed to the study design. L.M.L., D.A.E. and P.Y.W. conducted the study. L.M.L., D.A.E., C.M.B., R.G.H. and P.Y.W. contributed to data analysis. L.M.L., D.A.E., C.M.B., R.G.H., M.A.F. and P.Y.W. contributed to writing the paper. All authors have read and agreed to the published version of the manuscript.

Funding: This study was supported by internal institutional research funds from the Department of Anaesthesiology and Pain Medicine, University Hospital Bern, Bern, Switzerland; from the Burgergemeinde Bern (Reference number 2019-1055), and from an independent foundation, the "Stiftung für die Forschung in Anästhesiologie und Intensivmedizin" ("Foundation for Research in Anesthesiology and Intensive Care", 3010 Bern, [grant number 31/2019]). None of the three funding agencies had any role in the design and conduct of the study; collection, management, analysis, and interpretation of the data; preparation, review, or approval of the manuscript; or decision to submit the manuscript for publication.

Institutional Review Board Statement: Ethical approval for this study was provided by the Ethical Committee of the Canton Bern, Switzerland (KEK Bern, Project-ID 2018-01804, Chairperson Professor C. Seiler) on 3 December 2018.

Informed Consent Statement: Written informed consent was obtained from all subjects involved in the study.

Data Availability Statement: For confidentiality reasons, we are not able to make all data publicly available.

Acknowledgments: We thank Lukas Martig (Significantis GmbH, Bern, Switzerland), for conducting the statistical analyses.

Conflicts of Interest: The funders had no role in the design of the study; in the collection, analyses, or interpretation of data; in the writing of the manuscript, or in the decision to publish the results.

\section{Appendix A. Definitions of Postoperative Complications/Events}

\section{Appendix A.1. Gastrointestinal Complications}

- Ileus: no evidence of bowel function (no flatus and no passage of stool) with abdominal distension requiring cessation of oral intake and intravenous fluid support by POD 5;

- Constipation: no passage of stool without signs of ileus by POD 5;

- Gastric ulcer: diagnosis made by gastroscopy;

- Anastomotic bowel leak: considered as a complication if requiring surgery or prolonged drainage.

\section{Appendix A.2. Complications of Infections}

- Urinary tract infection: temperature $>38^{\circ} \mathrm{C}$ in the last $24 \mathrm{~h}$ and leukocytosis and a prompted urinary analysis that showed bacterial counts $>100,000$ requiring antibiotics; 
- Sepsis: bacterial infection and at least two of the following clinical signs: hypoor hyperthermia, tachycardia, tachypnea, leukocytopenia or leukocytosis, positive blood culture;

- Pneumonia: temperature $>38^{\circ} \mathrm{C}$ and leukocytosis and clinical signs of pneumonia, requiring antibiotics;

- Wound infection: pus can be expressed or aspirated, requiring surgical intervention.

\section{Appendix A.3. Wound Complications}

- Wound dehiscence: diagnosed clinically and requiring resuturing.

\section{Appendix A.4. Cardiac Events}

- Myocardial infarction: increase of the enzyme high-sensitive troponin $\mathrm{T}$ above the hospital laboratory's myocardial infarction threshold $(>0.05 \mu \mathrm{g} / \mathrm{L})$, and either new $\mathrm{Q}$ wave changes, or persistent changes in ST-T segments;

- Arrhythmia: confirmed by 12-lead electrocardiography and requiring new medication or electroconversion;

- Congestive heart failure and pulmonary edema: shortness of breath, rales, jugular venous distension, peripheral edema, third heart sound, radiologic signs (cardiomegaly, interstitial or alveolar edema), brain natriuretic peptide value $>500 \mathrm{pg} / \mathrm{mL}$ and diagnosis requiring diuretics;

- Transient brain natriuretic peptide increase: postoperative serum brain natriuretic peptide values between 100 and $500 \mathrm{pg} / \mathrm{mL}$ (considered as minor cardiac event).

\section{Appendix A.5. Thromboembolic Complications}

- Pulmonary embolism: evidenced by spiral computerized tomography scanning.

\section{Appendix A.6. Genitourinary Complications}

- Renal dysfunction: transient increase of creatinine: creatinine $>50 \%$ upper limit of normal value;

- Renal failure: severe reduction in glomerular filtration rate $\left(15-29 \mathrm{~mL} \cdot \mathrm{min}^{-1} \times 1.73 \mathrm{~m}^{-2}\right)$ at discharge;

- Urinary leakage: radiologically diagnosed, requiring stenting.

Appendix A.7. Neurological Complications

- Presence of a new focal deficit, confusion/delirium.

\section{References}

1. Apfel, C.C.; Meyer, A.; Orhan-Sungur, M.; Jalota, L.; Whelan, R.P.; Jukar-Rao, S. Supplemental intravenous crystalloids for the prevention of postoperative nausea and vomiting: Quantitative review. Br. J. Anaesth. 2012, 108, 893-902. [CrossRef]

2. Cheuvront, S.N.; Kenefick, R.W. Dehydration: Physiology, assessment, and performance effects. Compr. Physiol. 2014, 4, 257-285. [PubMed]

3. Casa, D.J.; Armstrong, L.E.; Hillman, S.K.; Montain, S.J.; Reiff, R.V.; Rich, B.S.; Roberts, W.O.; Stone, J.A. National athletic trainers' association position statement: Fluid replacement for athletes. J. Athl. Train. 2000, 35, 212-224. [PubMed]

4. Hahn, R.G.; Waldreus, N. An aggregate urine analysis tool to detect acute dehydration. Int. J. Sport Nutr. Exerc. Metab. 2013, 23, 303-311. [CrossRef]

5. Ylinenvaara, S.I.; Elisson, O.; Berg, K.; Zdolsek, J.H.; Krook, H.; Hahn, R.G. Preoperative urine-specific gravity and the incidence of complications after hip fracture surgery: A prospective, observational study. Eur. J. Anaesthesiol. 2014, 31, 85-90. [CrossRef]

6. Hahn, R.G.; Bahlmann, H.; Nilsson, L. Dehydration and fluid volume kinetics before major open abdominal surgery. Acta Anaesthesiol. Scand. 2014, 58, 1258-1266. [CrossRef]

7. Hahn, R.G. Renal injury during hip fracture surgery: An exploratory study. Anaesthesiol. Intensive Ther. 2015, 47, $284-290$. [CrossRef]

8. Wuethrich, P.Y.; Burkhard, F.C.; Thalmann, G.N.; Stueber, F.; Studer, U.E. Restrictive deferred hydration combined with preemptive norepinephrine infusion during radical cystectomy reduces postoperative complications and hospitalization time: A randomized clinical trial. Anesthesiology 2014, 120, 365-377. [CrossRef] 
9. Bleier, J.I.; Aarons, C.B. Perioperative fluid restriction. Clin. Colon. Rectal Surg. 2013, 26, 197-202.

10. McCaul, C.; Moran, C.; O'Cronin, D.; Naughton, F.; Geary, M.; Carton, E.; Gardiner, J. Intravenous fluid loading with or without supplementary dextrose does not prevent nausea, vomiting and pain after laparoscopy. Can. J. Anesth. 2003, 50, 440-444. [CrossRef]

11. Hahn, R.G.; Grankvist, N.; Krizhanovskii, C. Urinary analysis of fluid retention in the general population: A cross-sectional study. PLoS ONE 2016, 11, e0164152. [CrossRef]

12. Stark, P.A.; Myles, P.S.; Burke, J.A. Development and psychometric evaluation of a postoperative quality of recovery score: The, QoR-15. Anesthesiology 2013, 118, 1332-1340. [CrossRef]

13. Bennett-Guerrero, E.; Welsby, I.; Dunn, T.J.; Young, L.R.; Wahl, T.A.; Diers, T.L.; Phillips-Bute, B.G.; Newman, M.F.; Mythen, M.G. The use of a postoperative morbidity survey to evaluate patients with prolonged hospitalization after routine, moderate-risk, elective surgery. Anesth. Analg. 1999, 89, 514-519. [CrossRef]

14. Loffel, L.M.; Kleeb, B.; Burkhard, F.C.; Wuethrich, P.Y. Perioperative use of crystalloids in patients undergoing open radical cystectomy: Balanced, Ringer's maleate versus a glucose 5\%/potassium-based balanced solution: Study protocol for a randomized controlled trial. Trials 2014, 15, 1-8. [CrossRef]

15. Loffel, L.M.; Burkhard, F.C.; Takala, J.; Wuethrich, P.Y. Impact of a potassium-enriched, chloride-depleted 5\% glucose solution on gastrointestinal function after major abdominopelvic surgery: Sesults of a randomized controlled trial. Anesthesiology 2016, 125, 678-689. [CrossRef]

16. Gan, T.J.; Diemunsch, P.; Habib, A.S.; Kovac, A.; Kranke, P.; Meyer, T.A.; Watcha, M.; Chung, F.; Angus, S.A.A.-C.; Apfel, C.C.; et al Consensus guidelines for the management of postoperative nausea and vomiting. Anesth. Analg. 2014, 118, 85-113. [CrossRef] [PubMed]

17. Apfel, C.C.; Laara, E.; Koivuranta, M.; Greim, C.A.; Roewer, N. A simplified risk score for predicting postoperative nausea and vomiting: Conclusions from cross-validations between two centers. Anesthesiology 1999, 91, 693-700. [CrossRef]

18. Wuethrich, P.Y.; Burkhard, F.C. Improved perioperative outcome with norepinephrine and a restrictive fluid administration during open radical cystectomy and urinary diversion. Urol. Oncol. 2015, 33, 66.e21. [CrossRef]

19. Brunner, E.; Domhof, S.; Langer, F. Nonparametric, Analysis of Longitudinal, Data in Factorial, Experiments; Wiley: Hoboken, NJ, USA, 2002.

20. Arnaud, M.J. Mild dehydration: A risk factor of constipation? Eur. J. Clin. Nutr. 2003, 57 (Suppl. 2), S88-S95. [CrossRef]

21. Engel, D.; Loffel, L.M.; Wuethrich, P.Y.; Hahn, R.G. Preoperative concentrated urine increases the incidence of plasma creatinine elevation after major surgery. Front. Med. 2021, 8, 699969. [CrossRef] [PubMed]

22. Ekman, L.; Johnson, P.; Hahn, R.G. Signs of Dehydration after Hip, Fracture Surgery: An, Observational Descriptive, Study. Medicina 2020, 56, 361. [CrossRef]

23. Johnson, E.C.; Munoz, C.X.; Le Bellego, L.; Klein, A.; Casa, D.J.; Maresh, C.M.; Armstrong, L.E. Markers of the hydration process during fluid volume modification in women with habitual high or low daily fluid intakes. Eur. J. Appl. Physiol. 2015, 115, 1067-1074. [CrossRef]

24. Johnson, P.; Waldreus, N.; Hahn, R.G.; Stenstrom, H.; Sjostrand, F. Fluid retention index predicts the 30-day mortality in geriatric care. Scand. J. Clin. Lab. Investig. 2015, 75, 444-451. [CrossRef]

25. Hahn, R.G.; Zdolsek, M.; Hasselgren, E.; Zdolsek, J.; Bjorne, H. Fluid volume kinetics of $20 \%$ albumin. Br. J. Clin. Pharmacol. 2019, 85, 1303-1311. [CrossRef]

26. Li, Y.; He, R.; Ying, X.; Hahn, R.G. Dehydration, hemodynamics and fluid volume optimization after induction of general anesthesia. Clinics 2014, 69, 809-816. [CrossRef]

27. Brandstrup, B.; Møller, A.M. The, Challenge of Perioperative, Fluid Management in Elderly, Patients. Curr. Anesth. Rep. 2019, 9 , 406-413. [CrossRef]

28. Zarbock, A.; Koyner, J.L.; Hoste, E.A.J.; Kellum, J.A. Update on perioperative acute kidney injury. Anesth. Analg. 2018, 127, 1236-1245. [CrossRef]

29. Bernardi, M.H.; Ristl, R.; Neugebauer, T.; Hiesmayr, M.J.; Druml, W.; Lassnigg, A. Very early changes in serum creatinine are associated with 30-day mortality after cardiac surgery. Eur. J. Anaesthesiol. 2020, 37, 898-907. [CrossRef]

30. Furrer, M.A.; Schneider, M.P.; Löffel, L.M.; Buckhard, F.C.; Wuethrich, P.Y. Impact of intra-operative fluid and noradrenaline administration on early postoperative renal function after cystectomy and urinary diversion: A retrospective observational cohort study. Eur. J. Anaesthesiol. 2018, 35, 41-49. [CrossRef] [PubMed]

31. Loffel, L.M.; Bachmann, K.F.; Furrer, M.A.; Wuethrich, P.Y. Impact of intraoperative hypotension on early postoperative acute kidney injury in cystectomy patients-A retrospective cohort analysis. J. Clin. Anesth. 2020, 66, 109906. [CrossRef] 